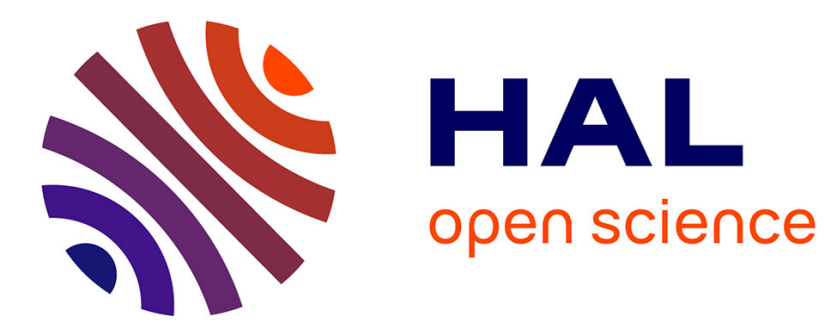

\title{
Spatial normalization of brain images and beyond
}

J.-F. Mangin, J. Lebenberg, S. Lefranc, N. Labra, Guillaume Auzias, M.

Labit, M. Guevara, H. Mohlberg, P. Roca, P. Guevara, et al.

\section{To cite this version:}

J.-F. Mangin, J. Lebenberg, S. Lefranc, N. Labra, Guillaume Auzias, et al.. Spatial normalization of brain images and beyond. Medical Image Analysis, 2016, 33, pp.127-133. 10.1016/j.media.2016.06.008 . hal-01464140

\section{HAL Id: hal-01464140 \\ https://hal-amu.archives-ouvertes.fr/hal-01464140}

Submitted on 16 Feb 2017

HAL is a multi-disciplinary open access archive for the deposit and dissemination of scientific research documents, whether they are published or not. The documents may come from teaching and research institutions in France or abroad, or from public or private research centers.
L'archive ouverte pluridisciplinaire HAL, est destinée au dépôt et à la diffusion de documents scientifiques de niveau recherche, publiés ou non, émanant des établissements d'enseignement et de recherche français ou étrangers, des laboratoires publics ou privés. 
Image Analysis

Elsevier Editorial System(tm) for Medical

Manuscript Draft

Manuscript Number:

Title: Spatial normalization of brain images and beyond

Article Type: SI: 20th anniversary of MedIA

Keywords: Spatial normalization

Cortical folding pattern

Diffeomorphism

Connectome

Corresponding Author: Dr Jean-François Mangin,

Corresponding Author's Institution: UNATI, Neurospin, CEA, Université Paris-Saclay

First Author: Jean-François Mangin

Order of Authors: Jean-François Mangin; Jessica Lebenberg; Sandrine Lefranc; Nicole Labra; Guillaume Auzias; Mickael Labit; Miguel Guevara; Hartmut Mohlberg; Pauline Roca; Pamela Guevara; Jessica Dubois; Francois Leroy; Ghislaine Dehaene-Lambertz; Arnaud Cachia; Timo Dickscheid; Olivier Coulon; Cyril Poupon; Denis Rivière; Katrin Amunts; Zhong Yi Sun

Abstract: The deformable atlas paradigm has been at the core of computational anatomy during the last two decades. Spatial normalization is the variant endowing the atlas with a coordinate system used for voxel-based aggregation of images across subjects and studies. This framework has largely contributed to the success of brain mapping. Brain spatial normalization, however, is still ill-posed because of the complexity of the human brain architecture and the lack of architectural landmarks in standard morphological MRI. Multi-atlas strategies have been developed during the last decade to overcome some difficulties in the context of segmentation. A new generation of registration algorithms embedding architectural features inferred for instance from diffusion or functional MRI is on the verge to improve the architectural value of spatial normalization. A better understanding of the architectural meaning of the cortical folding pattern will lead to use some sulci as complementary constraints. Improving the architectural compliance of spatial normalization may impose to relax the diffeomorphic constraint usually underlying atlas warping. Manifold learning could help to aggregate subjects according to their morphology. Connectivity-based strategies could emerge as an alternative to deformation-based alignment leading to match the connectomes of the subjects rather than images. 

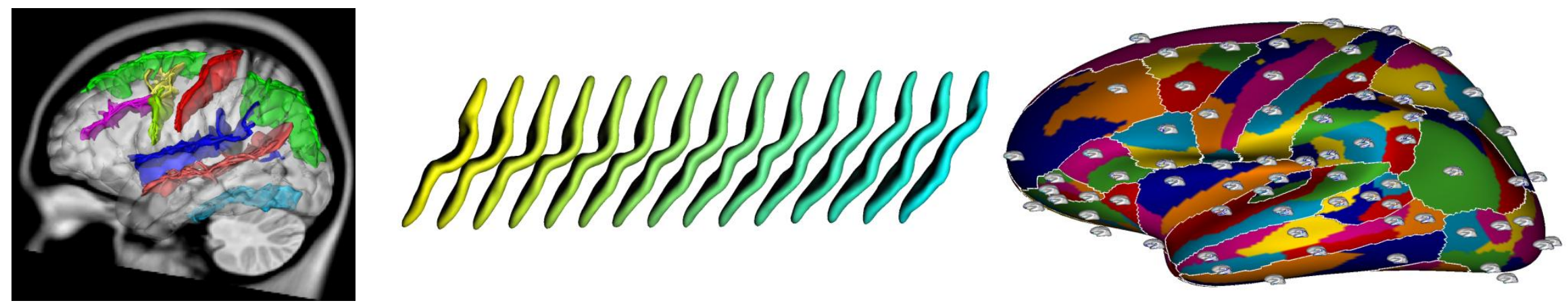


\section{*Highlights}

- Spatial normalization will have to evolve to improve its architectural compliance

- Specific research programs are required to select the sulci to be used during normalization

- Manifold learning is an efficient strategy to model the variability of the cortical folding pattern

- Connectivity-based parcellations will have a major impact on spatial normalization 


\section{Spatial normalization of brain images and beyond}

J.-F. Mangin ${ }^{1,9}$, J. Lebenberg ${ }^{1,6}$, S. Lefranc ${ }^{1,9}$, N. Labra ${ }^{1}$, G. Auzias $^{2}$, M. Labit ${ }^{1,9}$, M. Guevara ${ }^{3}$, H. Mohlberg ${ }^{4}$, P. Roca ${ }^{5}$, P. Guevara ${ }^{3}$, J. Dubois ${ }^{6}$, F. Leroy ${ }^{6}$, G. Dehaene-Lambertz ${ }^{6}$, A. Cachia ${ }^{8}$, T. Dickscheid $^{4}$, O. Coulon ${ }^{2}$, C. Poupon ${ }^{7,9}$, D. Rivière ${ }^{1,9}$, K. Amunts ${ }^{4}$, Z. Y. Sun ${ }^{1,9}$

1 : UNATI, Neurospin, CEA, Université Paris-Saclay, Gif-sur-Yvette, France

2 : Institut de Neurosciences de la Timone, UMR 7289, Aix Marseille Université, CNRS, Marseille, France

3: Dept. of Electr. Eng., Univ. de Concepcion, Concepcion, Chile

4: Institute of Neuroscience and Medicine (INM-1), Research Centre Jülich, 52425 Jülich, Germany

5 : Department of Neuroimaging, Sainte-Anne Hospital Center, Université Paris, Sorbonne Paris Cité, Center for Psychiatry \& Neurosciences, UMR 894 INSERM, Paris

6 : INSERM-CEA, Neurospin Center, Cognitive Neuroimaging Unit U992, Gif-Sur-Yvette, France

7 : UNIRS, Neurospin, CEA, Université Paris-Saclay, Gif-sur-Yvette, France

8 : CNRS UMR 8240, Laboratory for the Psychology of Child Development and Education, University Paris Descartes, Sorbonne Paris Cité, Paris, France

9 : CATI Multicenter Neuroimaging Platform, cati-neuroimaging.com, France

\section{Abstract}

The deformable atlas paradigm has been at the core of computational anatomy during the last two decades. Spatial normalization is the variant endowing the atlas with a coordinate system used for voxel-based aggregation of images across subjects and studies. This framework has largely contributed to the success of brain mapping. Brain spatial normalization, however, is still ill-posed because of the complexity of the human brain architecture and the lack of architectural landmarks in standard morphological MRI. Multiatlas strategies have been developed during the last decade to overcome some difficulties in the context of segmentation. A new generation of registration algorithms embedding architectural features inferred for instance from diffusion or functional MRI is on the verge to improve the architectural value of spatial normalization. A better understanding of the architectural meaning of the cortical folding pattern will lead to use some sulci as complementary constraints. Improving the architectural compliance of spatial normalization may impose to relax the diffeomorphic constraint usually underlying atlas warping. Manifold learning could help to aggregate subjects according to their morphology. Connectivity-based 
strategies could emerge as an alternative to deformation-based alignment leading to match the connectomes of the subjects rather than images.

\section{Convergence toward a standard brain warping strategy}

In the middle of the nineties, two different communities were setting off independently on a promising but difficult path aiming at comparing images of the brain through warping to a template. For the field of Medical Image Analysis, the goal was to adapt the atlases of medical textbooks to any anatomy (Miller et al., 1993). Practically, the methods proposed at that time often boiled down to warping manual segmentations of brain structures performed for a specific subject to any other subject. Then the template was simply the image of this specific subject endowed with properties ensuring smooth deformations, for instance using analogies with continuum mechanics. For the field of Brain Mapping, the goal was to perform voxel-based group analysis of low resolution functional PET images (Ashburner, 2012). The first methods were aligning brain images into a standardized coordinate system seeded by an old approach of stereotactic neurosurgery. In the context of the International Consortium for Brain Mapping (ICBM), the Montreal Neurological Institute (MNI) had proposed to build this system upon the average of 305 brains aligned using the landmark-based stereotactic strategy. Spatial normalization was achieved through automatic warping of any subject toward this average template. Warping was not regularized by a measure of roughness but by limiting the number of spatial basis functions used to model the deformations (SPM software). For both communities, spatial normalization was just one image registration problem among others.

Brain structure segmentation and coarse alignment of PET images are very different objectives, which could have led to very different research programs. The advent of FMRI, however, did bridge the gap and led to multiple cross-fertilizations. One decade later, one could speak of convergence into a single community acting under the umbrella called computational neuroanatomy. The intensive use of spatial normalization for $\mathrm{fMRI}$ group analysis and the advent of voxel-based morphometry had turn brain spatial normalization into a blockbuster technology (Ashburner, 2012). A myriad of publications have slowly converged toward a few general principles implemented by different software distributed throughout the brain imaging community. The ideal template is usually a populationaveraged image. The warping of individual subjects toward the template space is a smooth diffeomorphism preserving topology. Models of inter-subject variability, for instance "probabilistic" grey and white matter maps, contribute to improve the robustness of the normalization, usually in a Bayesian framework.

However, in spite of the sophistication and the robustness of the methods refined throughout the years, spatial normalization still looks more like an art than a scientific endeavor. The different software used throughout the community provide different results and these results depend on the chosen template and on various parameters intrinsic to the underlying registration methods. In the absence of consensual validations, evolving fashions 
help the users to choose the options that will be accepted by the reviewers. Everybody desperately needs brain normalization for brain mapping or biomarker research and everything is for the best in the best of worlds. Mesmerized by their outstanding research objectives, most of the users accept spatial normalization results as optimal for their purpose without questioning their weaknesses. As surprising as it may seem, users and designers often forget that the guideline for matching brains shall be architecture, because architecture stands as the topology of the underlying neural network. Provided that the brains to be matched come from the same species, this topology is supposed to be largely invariant across standard subjects at macroscopic scale. Simply speaking, the brain can be segregated into spatially localized entities (cortical areas, deep nuclei, etc.), each supposed to subserve a certain cognitive function within a network. The list of entities and their axonbased connections, called nowadays the connectome, is supposed to be species specific, with useful homologies across species.

\section{Forcing brain architecture into spatial normalization}

Brain architecture is amazingly complex and involves different notions from one part of the brain to the other. Contrary to some belief, the underlying research field is very active, building upon a continuous flow of new mapping technologies. For instance, Brodmann's mosaic-like maps in use in a lot of publications reflect an outdated vision of architectonic segregation of the cerebral cortex (Amunts and Zilles, 2015). Furthermore, the microscopic organization of the cortical mantle embeds cues like myelin or neurotransmitter density complementary to the cell type heterogeneities used by Brodmann and leading to additional macroscopic subdivisions. Connectivity, beyond reach before the advent of modern imaging modalities, has the potential to reveal further subdivisions inside areas homogeneous for microscopic cues. The meso-scale embeds various kinds of modular-like structures looking like column or blobs, often organized in maps like retinotopy or somatotopy. Note finally that for each architectural cue, the phenomenology of areal borders varies from abrupt to more gradual changes.

Unfortunately, the T1-weighted MR scans classically used to perform spatial normalization include very few architectural features. Regarding deep grey matter structures, the border of some nuclei can be visualized, but their subdivisions are out of reach, and numerous other nuclei, for instance in the brain stem, require other MR contrasts to be localized. White matter appears as a uniform compartment except for a few cues along large pathways like optic radiations. Fortunately, white matter bottlenecks provide a few important landmarks to be aligned across subjects like corpus callosum or anterior commissure. Regarding the cerebral cortex, the main architectural information included in these scans is thickness of the cortical mantle, but reaching specificity to cortical areas would require thickness of cortical layers, and the morphology of cortical folds. The situation of the cerebellum is even worst because of unsufficient spatial resolution. 
Since the link between the cortical folding pattern and architecture is unclear except in primary areas (Fischl et al., 2008), the earliest spatial normalization techniques did not really try to align cortical sulci. This choice resulted also from the difficulties raised by the large variability of the folding pattern across individuals. Hence, the first template brains driving the warping to the standard space were fuzzy brain images obtained by averaging a large number of normalized brain images without sulcus alignment. For the same reasons, the template brains used to adapt anatomical textbooks included only segmentations of deep nuclei and cortical lobes defined by major fissures. Nevertheless, during the following stages of algorithmic development, because of the lack of simple alternative, the quality of the matching of the folding patterns has often been used as a proxy of the quality of the architectural compliance of the normalization. Focusing on this target led to a major methodological fork: performing the normalization in a spherical 2D world corresponding to the topology of the cortical sheet (at least when connectivity is not taken into account). The resulting normalization tools (Freesurfer and Civet software) have rapidly achieved an impressive step forward with regard to alignment of the primary sulci, probably because of a large reduction of ambiguities in fold matching. But volumetric normalization tools have now probably caught up to a large extent with these surface-based approaches. The volumetric average brains computed after spatial normalization now include most of the large primary sulci. Sophisticated iterative normalization designs can even lead to quasi-realistic volumetric or surfacic average brains including representations of secondary sulci strikingly different from the idiosyncrasies of the individual brains (Fonov et al., 2011) (cf. Fig1). The future, pushed forward by the Human Connectome Project, http://www.humanconnectome.org/, is probably a hybrid world combining volume-based referentials dedicated to deep nuclei and surface-based referentials dedicated to the cortex and the cerebellum.

Nowadays, in a lot of research protocols, additional MRI modalities provide richer architectural cues than T1-weighted scans. Diffusion-weighted acquisitions open a large door on the white matter organization that could trigger a major shift in normalization strategies. Some tools provide already the possibility to align multivariate images based on the diffusion tensor (DTI-TK software). Innovative research programs aim at aligning tractograms or other representations of diffusion-based connectivity. T2-weigted acquisitions can yield maps of heavily myelinated grey matter regions, indicating specific architectural entities to be aligned across subjects. Advanced use of diffusion imaging provides complementary information about grey matter microstructure like neurite density that could help to localize some architectonic areas. T2*-weighted acquisitions can reveal a wide variety of architectural information at the level of deep nuclei, especially using high magnetic fields. Finally, functional MRI can provide a myriad of functionally relevant features, like resting state networks, sets of classical activations or study specific activations, and the inner organization of some functional areas. Hence, multimodal spatial normalization is a highly probable future (Robinson et al., 2014), which will require new standardization efforts. 
This promising future will capitalize upon the sharing of large databases of high-quality multimodal images through efforts such as the Human Connectome Project (HCP), or the European Connect Project (Assaf et al., 2013) (Archi database used in this paper's figures, to be distributed by the Human Brain Project, https://www.humanbrainproject.eu). Such databases include high quality diffusion acquisitions and rich functional localizers that will be used by the community to set-up the future normalization standards. For instance, alternative normalization strategies based on structural modalities ( $T 1, \mathrm{~T} 2$, diffusion) will be compared according to their capacity at aligning functional activations. It should be noted that the computational neuroanatomy community has always been missing such high quality individual activation datasets to pave the way toward objective validation criteria.

To complement the public datasets mentioned above allowing the study of interindividual variability, the Human Brain Project will bring to the community strategic architectural data like outstanding microscopy-based postmortem datasets and massive in vivo acquisitions performed for a few subjects. For instance, the "bigbrains" will convey whole brain microscopic descriptions of cell spatial organization to be used to revisit cytoarchitectony (Amunts et al., 2013). Polarized Light Imaging will generate whole brain microscopic descriptions of axon orientations (Axer et al., 2011). The IBC project of HBP aims at obtaining more than 50 hours of $\mathrm{fMRI}$ acquisitions for each subject, in order to aggregate activation protocols designed across the world (https://github.com/hbp-brain-charting). The complementary Biquette project will accumulate diffusion-based acquisitions to push the limits of in vivo microstructure mapping on the same subjects as IBC. All these datasets will be combined into a multi-resolution atlas of the Human brain architecture embedded into the standard spaces of the brain mapping communities. This atlas will be used as a scaffold to help the neuroscience communities to aggregate data into the HBP neuroinformatics platform.

\section{The cortical folding pattern}

While the number of architectural cues used to spatially normalized brain images is bound to increase, the variability of the cortical folding pattern will remain an issue. Provided that we improve our understanding of the link between architecture and the folding process, the folding pattern could be better exploited during spatial normalization. Folding is often reduced to an evolutionary trick increasing the surface area of the cortex without impacting the skull volume. From this point of view, the detailed topography of the folding pattern is meaningless. The essence of the folding pattern, however, emerges during the last trimester of fetal life, a key time window for the architectural development of the cortical sheet (architectonic arealisation and axonal connectivity establishment). The forces driving the folding dynamics are still unclear but several recent hypotheses assume interactions with the primal sketch of the cortical architecture. Hence, the inter-individual variability of the gross sulcal patterns probably provides a proxy to the variability of the cortex architecture. 
Surprisingly, the anatomical literature is rather sparse of rules to overcome the variability of the cortical sulci topography across individuals. Long primary sulci can be split into pieces by annectant gyri. These pieces can be recombined in non standard ways to create unusual folding patterns without clear match with the anatomical nomenclature. Furthermore, primary sulci are surrounded by numerous shallower folds increasing the complexity to be decrypted. Whatever the progresses achieved by modern normalization tools, imposing explicitly sulcus-based constraints will probably be useful to deal safely with some specific situations. Thanks to dedicated computer vision approaches (Mangin et al., 2015b), it is now possible to define automatically more than 120 cortical sulci (Perrot et al., 2011) in order to provide them to sulcus-based normalization frameworks (Auzias et al., 2011; Auzias et al., 2013). The automatic recognition process is not error prone and can be questioned, but once the folds have been extracted using a computational technique, they can be matched manually to any sulcus nomenclature in a reasonable time, according to the needs. Sulcusbased constraints can be used to get robust normalization of brains with unusual folding pattern but also of images with unusual contrasts disturbing standard normalization techniques (premature babies, fetus, infants during the myelination process, postmortem histological 3D reconstruction, cf Fig1).

What are the sulci to be aligned across brains? While the interest of imposing the alignment of sulci in primary areas has been proven (Fischl et al., 2008), the question is largely opened elsewhere. In non primary areas, the number of studies reporting strong links between the folding pattern and functional or architectonic maps is very low. In our opinion, this global lack of knowledge reveals the difficulty of this kind of investigations more than the absence of link. Recent hypotheses to explain the nature of the folding process often imply such links. For instance, it is now relatively consensual that a protomap of the primary folding pattern exists at the level of the subventricular zone, before the neuronal migrations leading to the construction of the cortical mantle (de Juan Romero et al., 2015). This protomap, supposed to result from heterogeneous gene expressions maybe related to morphogen gradients, could be the primal sketch of the differentiation of the cortex into architectonic areas but also of the primary folding pattern. It was shown recently that realistic folding geometry could be obtained from simulations of the mechanical instability driven by tangential expansion of the gray matter constrained by the white matter (Tallinen et al., 2014). Since progyral areas of the protomap correspond to increased multiplication of neurons, they may correspond to expansion maxima responsible for selecting the primary attractor of the folding dynamics. Slight tensions stemming from the fiber tracts could drive the following dynamic steps and shape the secondary and tertiary folds.

Future work will lead to assess one by one the added value of each sulcus in the normalization process, relative to its capacity at improving the alignment of architectural data like postmortem architectonic maps or in vivo fMRI maps (Mangin et al., 2015a). In a context where the amount of architectural information planned to be used for normalization is rapidly increasing, it would be important to discard the sulci generating misleading implicit 
or explicit constraints. A seminal work in this direction has been achieved using machine learning techniques to tune the influence of the different folds during surface-based normalization (Yeo et al., 2010).

\section{The diffeomorphism delusion}

We do not have enough genes to code for all the complexity of our brain architecture. Several mechanisms responsible for this architecture build upon generative rules rather than upon genetically-controlled maps. For instance, infant brain growth results in an overabundance of connections, which are removed later to ensure that a functional organization of circuitry is established. While genetics could control the species-specific primary cortical folding pattern, a large part of the variability of the secondary folding pattern could simply result from the chaotic nature of the folding dynamics. Nevertheless, as mentioned above, subject-specific architectonic or connectivity-based features could select this secondary folding pattern in a way or another. An even bolder hypothesis consists in thinking that nature could recycle random secondary folding pattern to derive an optimal placement of cortical areas (Foubet and Toro, 2016).

Baring in mind these few ideas about development, it is easy to understand why there is no Darwinian pressure on the existence of an architecturally compliant diffeomorphism between pairs of brains of the same species (see Fig 2). The connectome-based point of view is stressing the fact that the brain organization relies on a topological model rather than on the spatial embedding of the underlying graph. Hence we should prepare for a future where diffeomorphism-based normalization is a useful tool but not an end. It should be noted that for segmentation purpose, the community has now realize that a multi-atlas strategy is much more efficient than a single atlas strategy. This discovery has led to a massive divergence from the spatial normalization paradigm. The segmentation of a specific subject stems from the fusion of the most similar atlases according to alignment quality. In this context, the normalization paradigm is fading away: the resulting segmentation is not stuck to a standard coordinate system, because the detailed alignment of the different atlases to such a system is out of the scope. Patch-based methods, the most recent segmentation strategy, go even one step further. They achieve segmentation from the computation of patch-based similarities that do not require global alignment between the subject and the atlases.

The pragmatic move of the segmentation community away from sophisticated normalization-based strategies stems from the realization that the alignment of brains with very different shapes remains ill-posed whatever the a priori knowledge used to regularize the problem. While this observation is relatively intuitive regarding the cortical folding patterns and their disturbing variability, the successes of the multi-atlas strategy seem to prove that the situation is similar for simpler brain structures. The alignment of a specific sulcus across a group of subjects raises a lot of issues related to its variable interruptions and branches (Mangin et al., 2015a). Since registration techniques provide more meaningful 
alignment when dealing with similar patterns, normalization should be applied independently in subgroups of subjects with the same sulcus pattern, leading to several independent standard spaces. Performing independent group analysis in each of these spaces would lead to pattern-specific brain mapping. Provided that the sparse databases of histology-based individual architectural maps include each of the sulcus patterns of interest, a second stage of meta-alignment informed by architecture could be developed to bridge the gaps between these pattern-specific standard spaces. Rich multimodal MRI datasets could also contribute to the meta-alignment to complement histology-based databases. Note again that this meta-alignment stage may have to give up on using the usual diffeomorphism framework whenever different morphological patterns reveal non compatible spatial embedding of the underlying architecture.

Applying this two level strategy requires the definition of patterns in the spirit of what was done for 25 brains in the atlas of Ono. Automatic data mining techniques have been designed to extend the work of Ono to larger datasets in order to obtain a reliable estimation of frequent sulcal patterns (Sun et al., 2009). Applying non supervised clustering to thousand of brains, they aim at providing an exhaustive dictionary of templates of sulcal patterns to be used when designing the multi-pattern normalization strategy mentioned above. Several complex issues have to be addressed. First, what should be the optimal scale for these local templates of folding patterns? Ambiguities in sulcus recognition lead to split the global folding pattern into groups of sulci minimizing intergroup mismatch rather than dealing with sulci one by one. Second, once local templates of patterns and architecturally compliant alignments between them have been defined, how to build a global standard space?

The concept of sulcal pattern is relatively easy to understand: for instance, the central sulcus exists either as one single continuous furrow, or as a split furrow in case of interruption at the level of the middle fronto-parietal "pli de passage", a transverse usually buried gyrus (Mangin et al., 2015a). In practice, clustering techniques are challenged because of the existence of continuous variations from one pattern to the other. With regard to the central sulcus interruption, for instance, the "pli de passage" can be more or less buried, leading to a shape continuum between the interrupted configuration and the configuration with a very deep "pli de passage". Hence, defining the optimal number of patterns is difficult. As an alternative, it was proposed to capture a low dimensional manifold approximation of the high dimensional space spanned by each sulcus (Sun et al., 2012). The pattern-based brain mapping strategy mentioned above has been directly extended to this manifold strategy as illustrated in Figure 3 (Sun et al., 2015). The manifold approach refines the multi-pattern strategy because it helps to understand the transformations of the architectural templates along with the geodesic transformations of the morphological patterns, providing a guideline for the second level alignment. Whenever a diffeomorphism can transform two patterns into one another, the manifold-based geodesic is probably the candidate with highest architectural likelihood, because it is based on a chain of pairwise registrations of similar 
morphologies. In return, when developmental phenomena forbid any diffeomorphism to align the two architectural patterns, the manifold is not simply connected and other strategies will have to be designed, looking like alchemical transmutations at our current level of understanding.

\section{The connectome Graal}

Historically, experts of the brain architecture have been relying on mosaic-like parcellations of grey matter to represent maps of the brain (Amunts and Zilles, 2015). Two decades ago, hardly anything was known about long-distance pathways in the human brain, other than the general distribution of major fiber tracts discernible from classical blunt dissection studies (Van Essen et al., 2016). The explosion of methods allowing connectivity mapping has been dramatic but so have the many challenges of carefully interpreting these highly complex datasets. Resting state functional MRI and diffusion-based MRI have now the potential to provide architectural parcellations of the entire brain at the group level (cf Fig. 4). The outstanding datasets distributed by the Human Connectome Project will probably allow similar parcellations at the individual levels. Such an achievement will probably largely supersede the current spatial normalization strategy, provided that routine acquisitions can rapidly catch up with the HCP data quality.

\section{Acknowledgement}

This work was partially funded by the French National Agency for Research (ANR12JS0300101 'MODEGY', ANR14CE300014 'APEX') and the European FET Flagship project 'Human Brain Project' (SP2) FP7-ICT-2013-FET-F/604102. The "CONNECT/Archi" Database" is the property of the CEA NeuroSpin centre, was designed under the supervision of Dr. Cyril Poupon and Dr. Jean-François Mangin, and was funded by the Federative Research Institute 49, the HIPPIP European grant, and the European CONNECT project (http://www.brain-connect.eu). Acquisitions were performed by the scientists involved in the Multiscale Brain Architecture research program of NeuroSpin and by the staff of the UNIACT Laboratory of NeuroSpin (headed by Dr. Lucie Hertz-Pannier), under the ethical approval CPP100002/CPP100022 (principal investigator Dr. Denis Le Bihan). Pending the availability of the HBP neuroinformatics platform, access to the database can be requested from cyril.poupon@cea.fr.

\section{Figure Captions}

Fig1: Sulcus-based alignment of non standard images: Groupwise diffeomorphic registration was achieved using DISCO (Auzias et al., 2011), and can be used to initialize an image-based deformation framework for further improvement. DISCO is embedded into BrainVISA (http://brainvisa.info). The Morphologist pipeline of BrainVISA dedicated to sulcus 
extraction has been adapted to fetus, infants and histological data. The adult template is ICBM 2009c Nonlinear Asymmetric (Fonov et al., 2011).

Fig2: Sulci and U-fiber bundles of the frontal lobe: Sulci were defined automatically using Morphologist, a dedicated pipeline of BrainVISA (Perrot et al., 2011). U-fiber bundles were defined automatically using a refinement of the method described in (Guevara et al., 2012). Some inconsistencies across the 3 subjects result from sulcus or fiber labeling mistakes. However, the chances to find a labeling compatible with the existence of pairwise diffeomorphisms matching architectures are low. Function and topology can be two different stories as illustrated by chair design.

Fig3: One dimensional approximation of the central sulcus manifold: the manifold is inferred with Isomap algorithm from a pairwise similarity matrix (Sun et al., 2012). The one dimension approximation encodes the transition from a single knob configuration to a double knob configuration. FMRI group analysis performed along the manifold using a moving average strategy confirms that the upper knob is associated to the hand (Sun et al., 2015). In return, the reading-related premotor activation localization relative to sulci depends on the morphology of the central sulcus.

Fig4: Group-wise parcellation of the cortical surface from 79 subjects: Each gyrus of a freesurfer segmentation is split into clusters with homogeneous connectivity profiles defined from diffusion-based tractography after population averaging (Lefranc et al., 2016). Interindividual parcellations were obtained using a concatenation of the data rather than averaging (Roca et al., 2010).

\section{Bibliography}

Amunts K, Zilles K (2015) Architectonic Mapping of the Human Brain beyond Brodmann. Neuron 88:1086-1107.

Amunts K, Lepage C, Borgeat L, Mohlberg H, Dickscheid T, Rousseau M, Bludau S, Bazin PL, Lewis LB, Oros-Peusquens AM, Shah NJ, Lippert T, Zilles K, Evans AC (2013) BigBrain: an ultrahighresolution 3D human brain model. Science 340:1472-1475.

Ashburner J (2012) SPM: a history. Neuroimage 62:791-800.

Assaf $Y$ et al. (2013) The CONNECT project: Combining macro- and micro-structure. Neuroimage 80:273-282.

Auzias G, Colliot O, Glaunès JA, Perrot M, Mangin JF, Trouvé A, Baillet S (2011) Diffeomorphic brain registration under exhaustive sulcal constraints. IEEE Trans Med Imaging 30:1214-1227.

Auzias G, Lefèvre J, Le Troter A, Fischer C, Perrot M, Régis J, Coulon O (2013) Model-driven harmonic parameterization of the cortical surface: HIP-HOP. IEEE Trans Med Imaging 32:873-887.

Axer M, Amunts K, Grässel D, Palm C, Dammers J, Axer H, Pietrzyk U, Zilles K (2011) A novel approach to the human connectome: ultra-high resolution mapping of fiber tracts in the brain. Neuroimage 54:1091-1101.

de Juan Romero C, Bruder C, Tomasello U, Sanz-Anquela JM, Borrell V (2015) Discrete domains of gene expression in germinal layers distinguish the development of gyrencephaly. EMBO J 34:1859-1874. 
Fischl B, Rajendran N, Busa E, Augustinack J, Hinds O, Yeo BT, Mohlberg H, Amunts K, Zilles K (2008) Cortical folding patterns and predicting cytoarchitecture. Cereb Cortex 18:1973-1980.

Fonov V, Evans AC, Botteron K, Almli CR, McKinstry RC, Collins DL, Group BDC (2011) Unbiased average age-appropriate atlases for pediatric studies. Neuroimage 54:313-327.

Foubet $\mathrm{O}$, Toro R (2016) Mechanical morphogenesis and the development of neocortical organisation. http://dxdoiorg/101101/021311.

Guevara P, Duclap D, Poupon C, Marrakchi-Kacem L, Fillard P, Le Bihan D, Leboyer M, Houenou J, Mangin JF (2012) Automatic fiber bundle segmentation in massive tractography datasets using a multi-subject bundle atlas. Neuroimage 61:1083-1099.

Lefranc S, Roca P, Perrot M, Poupon C, Le Bihan D, Mangin JF, Rivière D (2016) Groupwise connectivity-based parcellation of the whole human cortical surface using watershed-driven dimension reduction. Med Image Anal 30:11-29.

Mangin J-F, Auzias G, Coulon O, Sun ZY, Rivière D, Régis J (2015a) Sulci as Landmarks. In: Brain Mapping: An Encyclopedic Reference (Arthur W. Toga, ed), pp 45-52: Academic Press: Elsevier.

Mangin J-F, Perrot M, Operto G, Cachia A, Fischer C, Lefèvre J, Rivière D (2015b) Sulcus Identification and Labeling. In: Brain Mapping: An Encyclopedic Reference (Toga AW, ed), pp 365-371: Academic Press: Elsevier

Miller MI, Christensen GE, Amit Y, Grenander U (1993) Mathematical textbook of deformable neuroanatomies. Proc Natl Acad Sci U S A 90:11944-11948.

Perrot M, Rivière D, Mangin JF (2011) Cortical sulci recognition and spatial normalization. Med Image Anal 15:529-550.

Robinson EC, Jbabdi S, Glasserlabel MF, Andersson J, Burgess GC, Harms MP, Smith SM, Van Essen DC, Jenkinson M (2014) MSM: A new flexible framework for Multimodal Surface Matching. Neuroimage.

Roca P, Tucholka A, Rivière D, Guevara P, Poupon C, Mangin JF (2010) Inter-subject connectivitybased parcellation of a patch of cerebral cortex. Med Image Comput Comput Assist Interv 13:347-354.

Sun Z, Perrot M, Tucholka A, Rivière D, Mangin J-F (2009) Constructing a dictionary of human brain folding patterns. Medical Image Computing and Computer-Assisted Intervention-MICCAI 2009:117-124.

Sun ZY, Pinel P, Rivière D, Moreno A, Dehaene S, Mangin JF (2015) Linking morphological and functional variability in hand movement and silent reading. Brain Struct Funct.

Sun ZY, Klöppel S, Rivière D, Perrot M, Frackowiak R, Siebner H, Mangin J-F (2012) The effect of handedness on the shape of the central sulcus. Neuroimage 60:332-339.

Tallinen T, Chung JY, Biggins JS, Mahadevan L (2014) Gyrification from constrained cortical expansion. Proc Natl Acad Sci U S A 111:12667-12672.

Van Essen DC, Donahue C, Dierker DL, Glasser MF (2016) Parcellations and Connectivity Patterns in Human and Macaque Cerebral Cortex In: Micro-, Meso- and Macro-Connectomics of the Brain, Research and Perspectives in Neurosciences (H. Kennedy, ed), pp 89-106.

Yeo BT, Sabuncu MR, Vercauteren T, Holt DJ, Amunts K, Zilles K, Golland P, Fischl B (2010) Learning task-optimal registration cost functions for localizing cytoarchitecture and function in the cerebral cortex. IEEE Trans Med Imaging 29:1424-1441. 

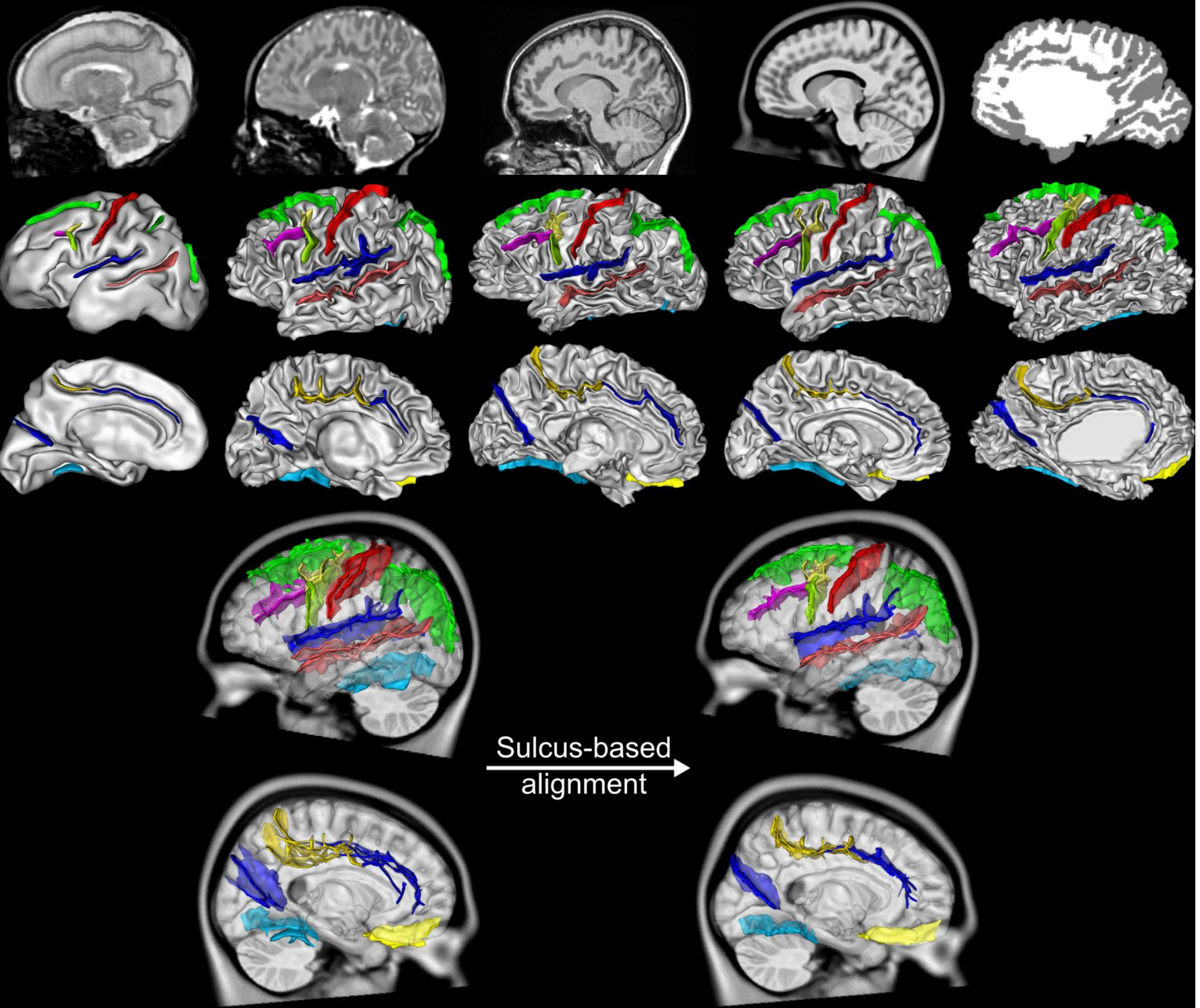

\section{Sulcus-based

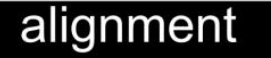

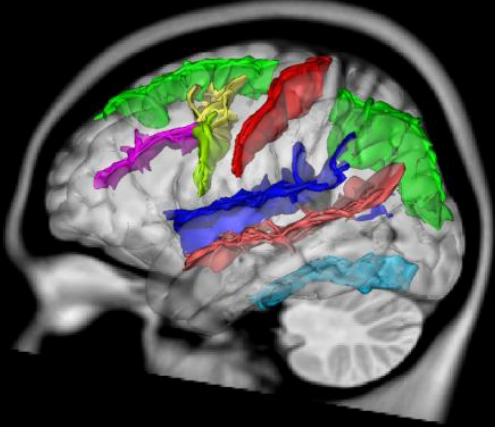

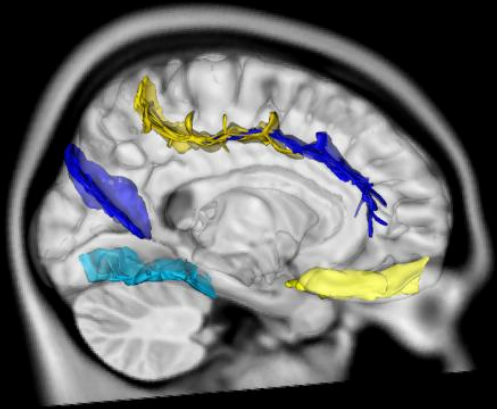



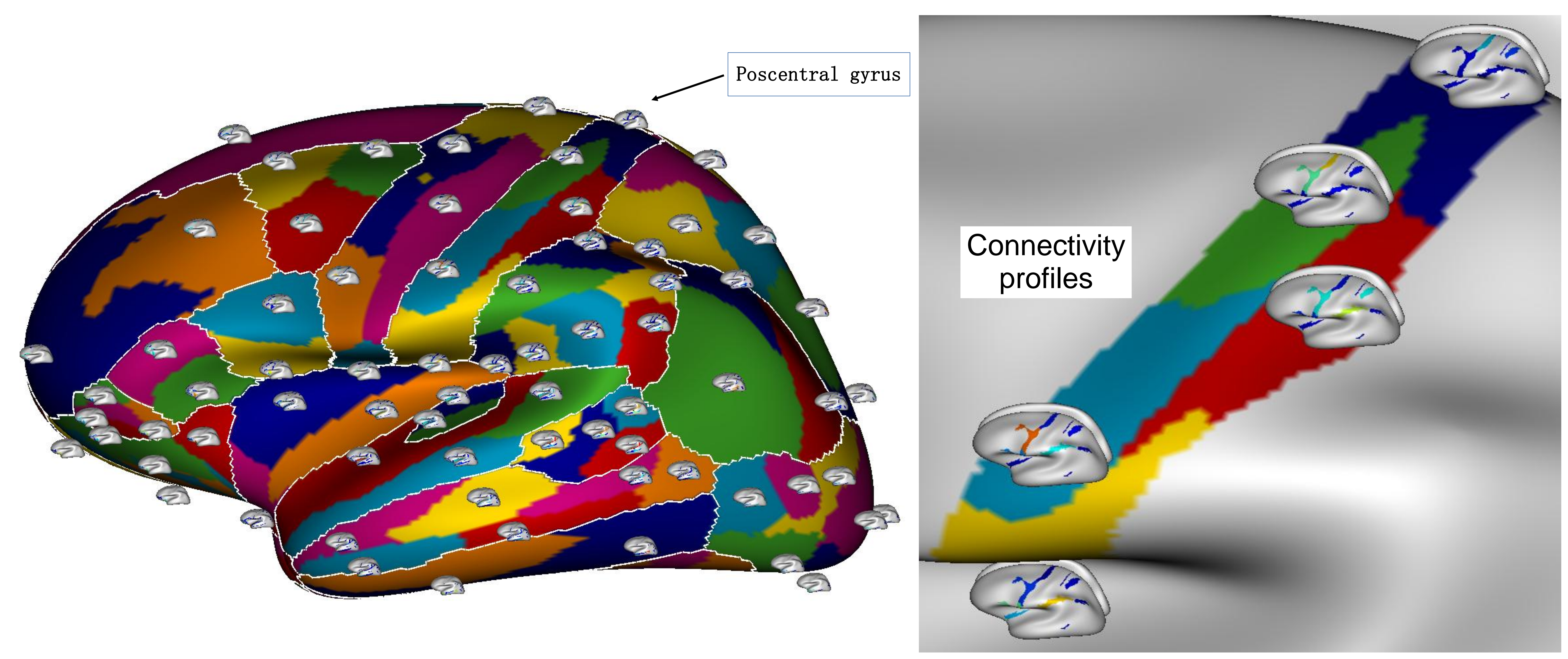

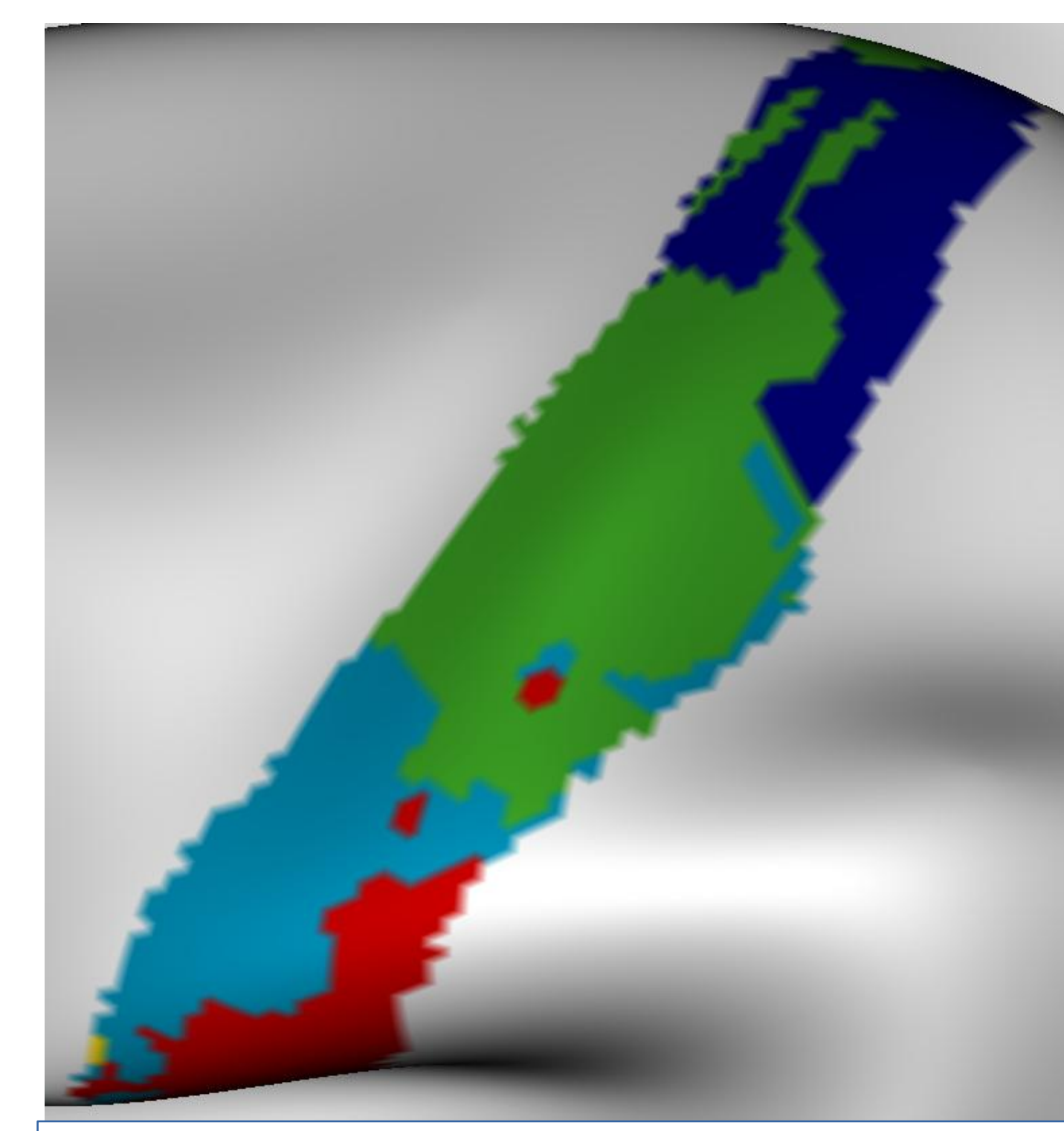

Subject 1

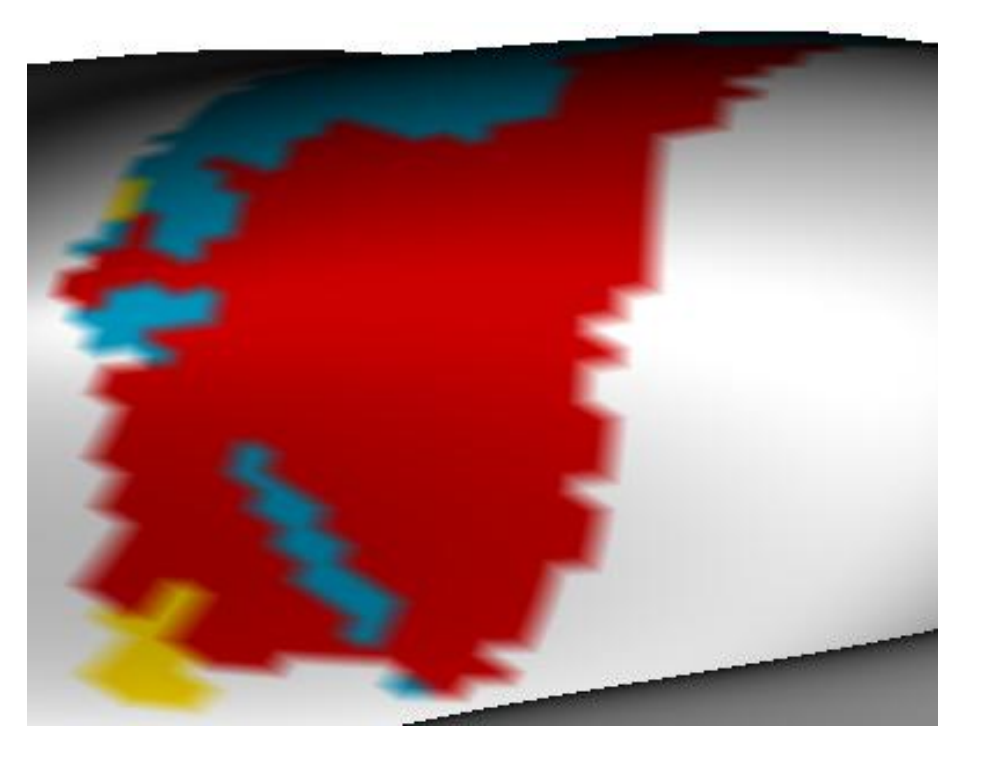

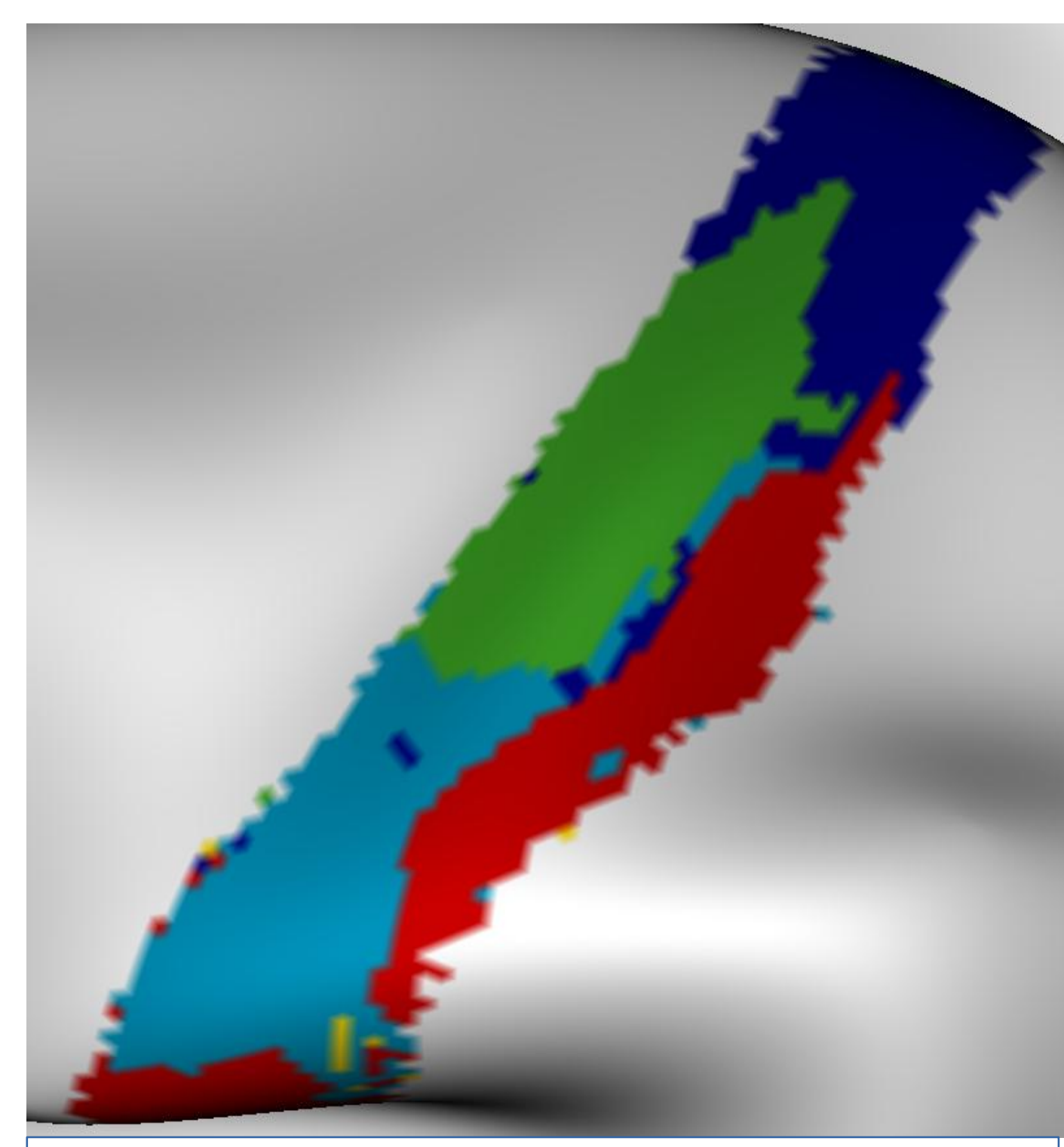

Subject 2

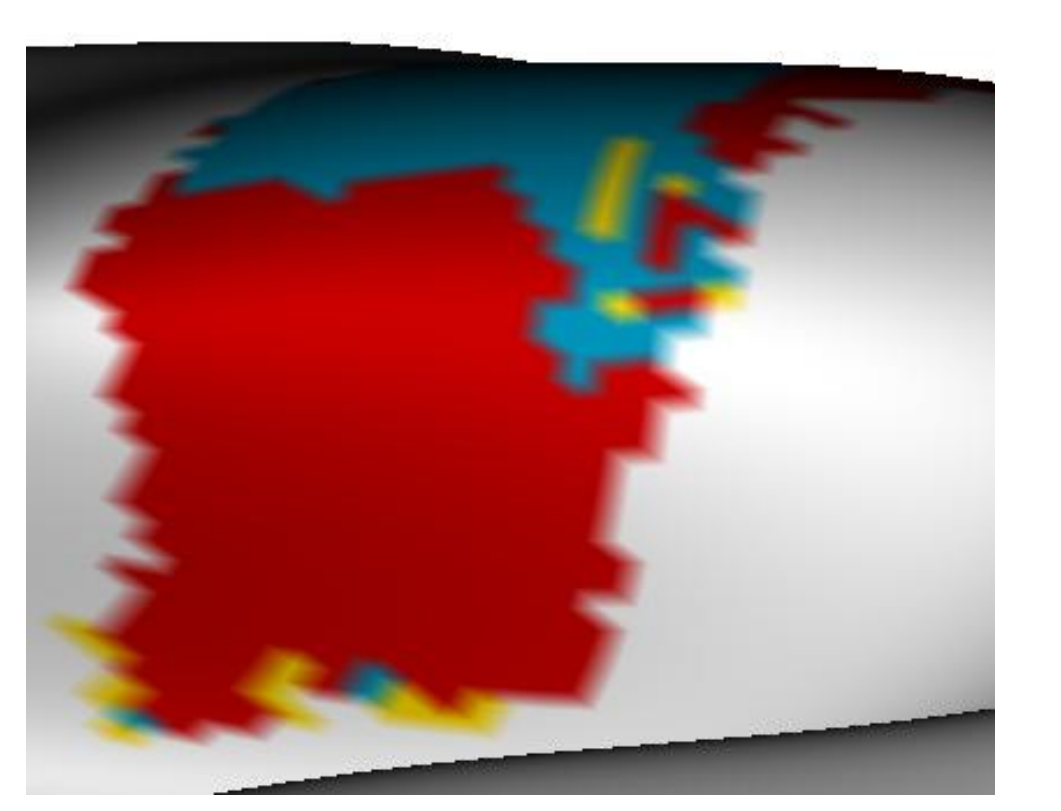

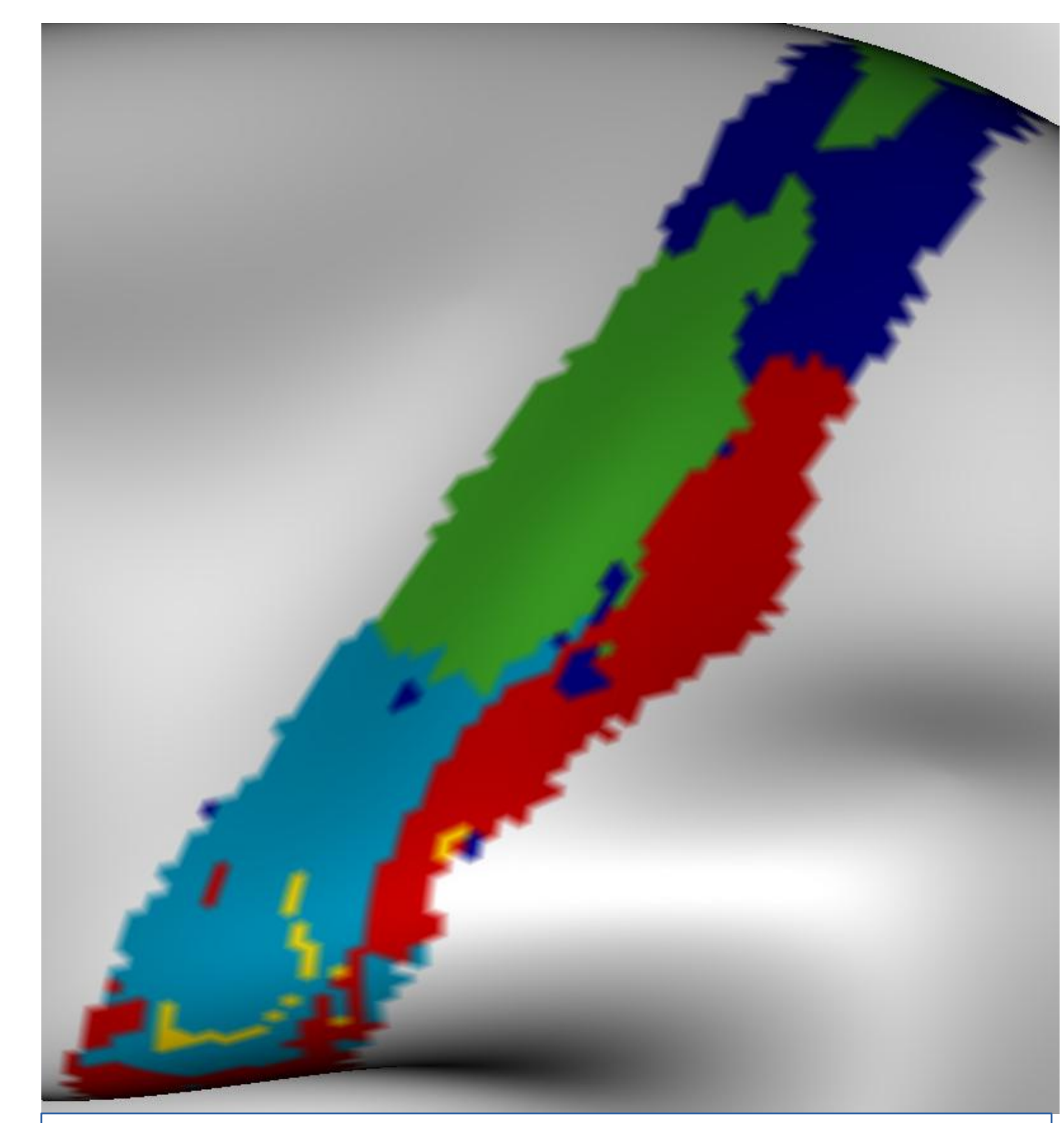

Subject 3

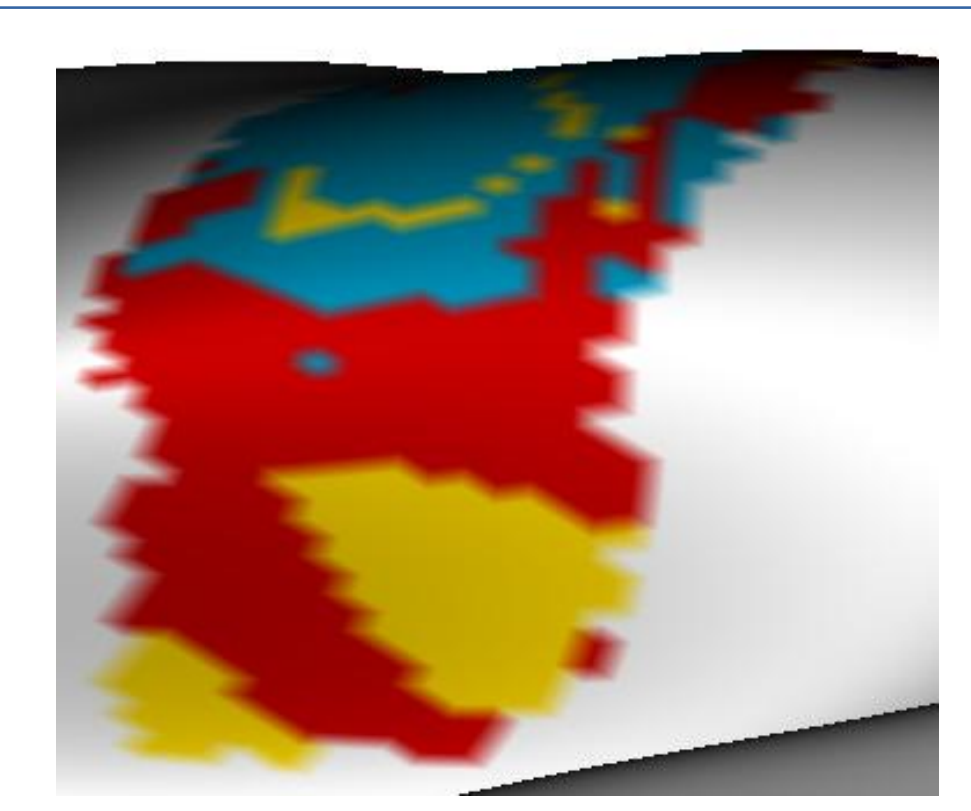

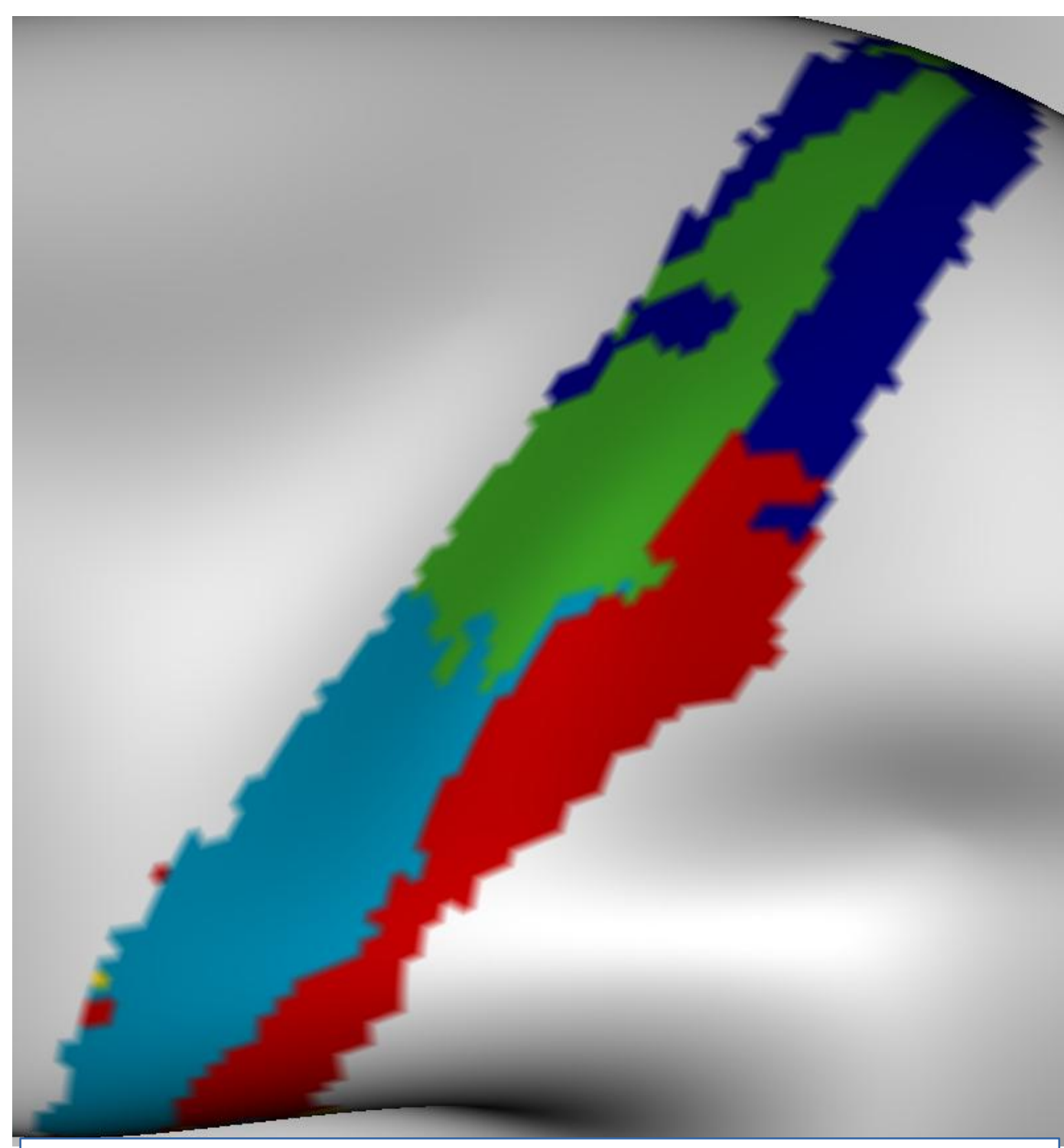

Subject 4

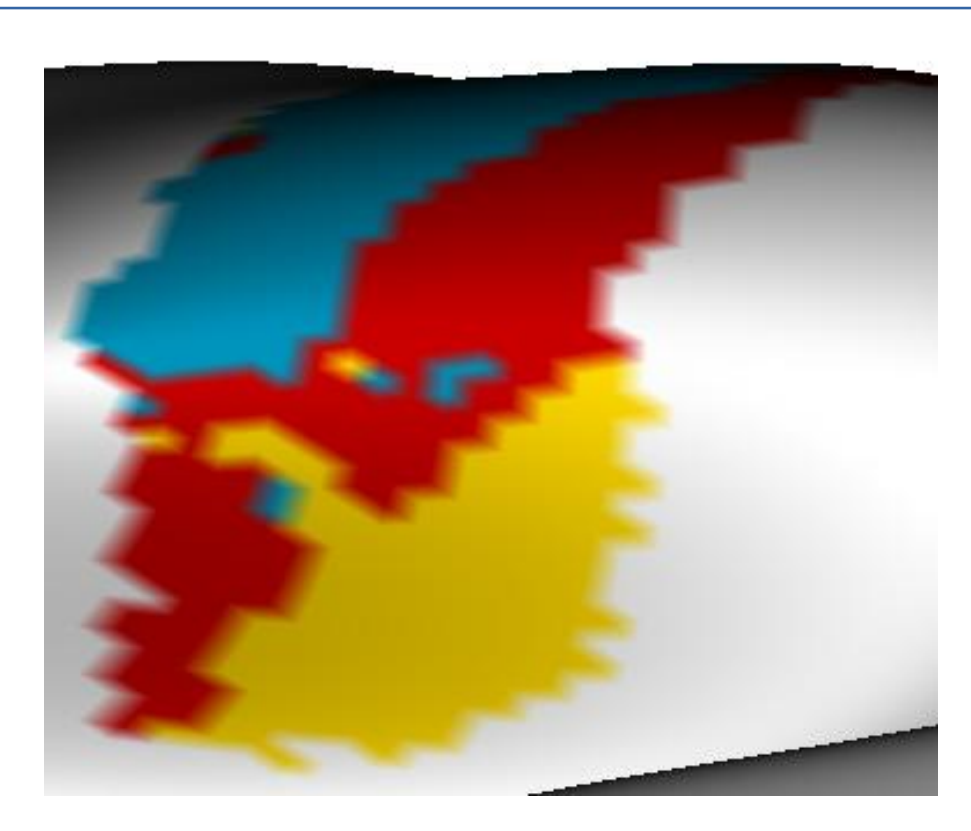

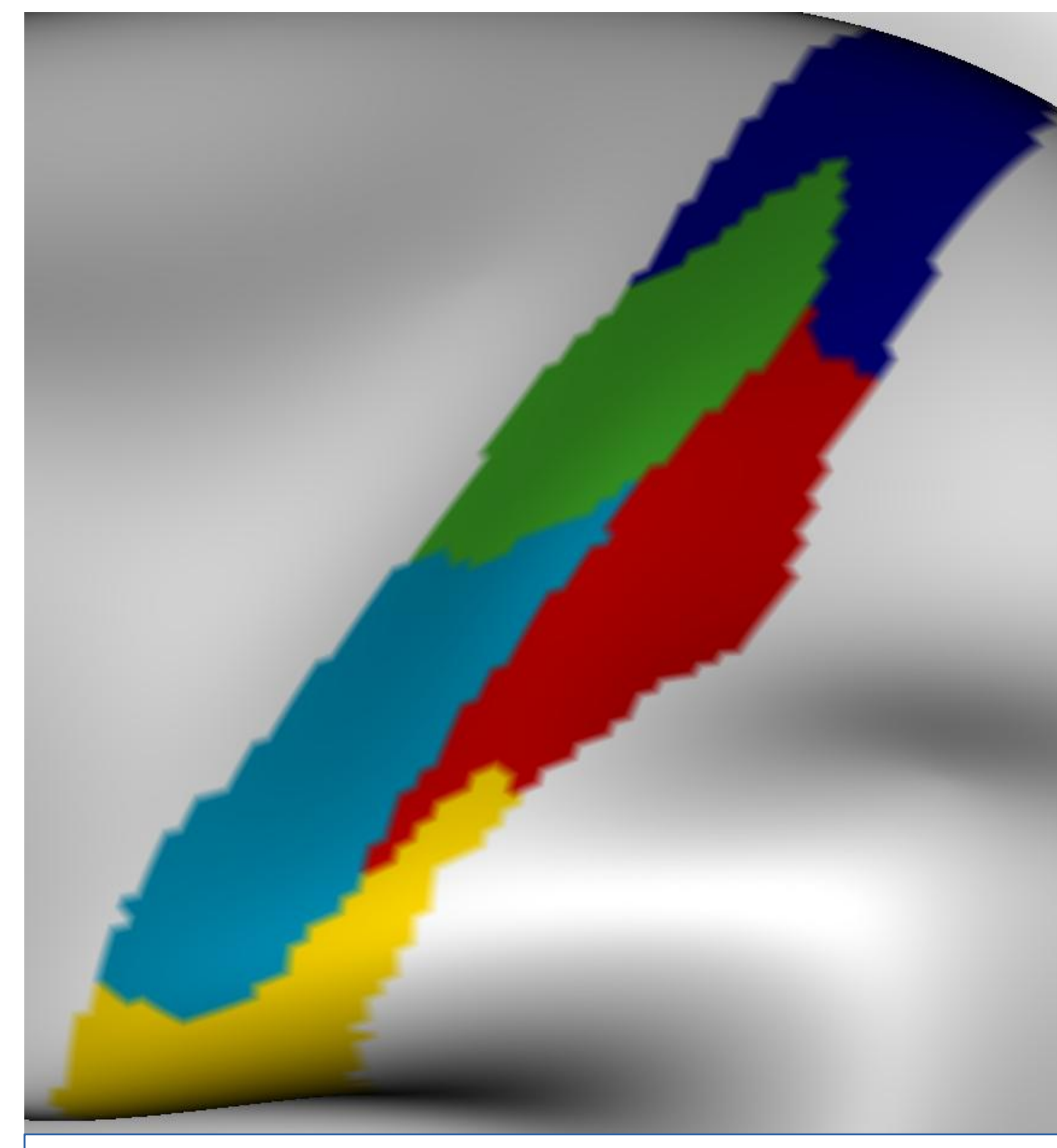

Average subject

Lower part of the gyrus 